Elena García-González, Maite Aramendía, Ricardo González-Tarancón, Naiara Romero-Sánchez and Luis Rello*

\title{
Detecting paraprotein interference on a direct bilirubin assay by reviewing the photometric reaction data
}

DOI 10.1515/cclm-2016-0690

Received August 4, 2016; accepted November 14, 2016

\section{Abstract}

Background: The direct bilirubin (D-Bil) assay on the AU Beckman Coulter instrumentation can be interfered by paraproteins, which may result in spurious D-Bil results. In a previous work, we took advantage of this fact to detect this interference, thus helping with the identification of patients with unsuspected monoclonal gammopathies. In this work, we investigate the possibility to detect interference based on the review of the photometric reactions, regardless of the D-Bil result.

Methods: The D-Bil assay was carried out in a set of 2164 samples. It included a group of 164 samples with paraproteins (67 of which caused interference on the assay), as well as different groups of samples for which high absorbance background readings could also be expected (i.e. hemolyzed, lipemic, or icteric samples). Photometric reaction data were reviewed and receiver operating characteristics (ROC) curves were used to establish a cut-off for absorbance that best discriminates interference.

Results: The best cut-off was 0.0100 for the absorbance at the first photometric point of the complementary wavelength in the blank cuvette. Once the optimal cut-off for probable interference was selected, all samples analyzed in our laboratory that provided absorbance values above this cut-off were further investigated to try to discover paraproteins. During a period of 6 months, we detected 44 samples containing paraproteins, five of which belonged to patients with non-diagnosed monoclonal gammopathies.

*Corresponding author: Luis Rello, Department of Clinical Biochemistry, Hospital Universitario “Miguel Servet”, Paseo Isabel La Católica 1-3, 50009 Zaragoza, Spain, Phone: +34-976765544, Fax: +34-976765543, E-mail: Irello@salud.aragon.es Elena García-González, Ricardo González-Tarancón and Naiara Romero-Sánchez: Department of Clinical Biochemistry, Hospital Universitario “Miguel Servet”, Zaragoza, Spain

Maite Aramendía: Centro Universitario de la Defensa-Academia General Militar de Zaragoza, Zaragoza, Spain
Conclusions: Review of the photometric reaction data permits the systematic detection of paraprotein interference on the D-Bil AU assay, even for samples for which reasonable results are obtained.

Keywords: direct bilirubin; interference; paraprotein; photometric reaction.

\section{Introduction}

Paraproteins are a common cause of interference in routine chemistry methods $[1,2]$, and the direct bilirubin (D-Bil) AU Beckman Coulter assay seems to be particularly sensitive to this interference [3-6]. This test is a two-cuvette (blank and color) endpoint assay based on the formation of azobilirubin [by reaction of conjugated (direct) bilirubin from the serum sample with a diazonium salt at low $\mathrm{pH}$, which is then measured bichromatically at 570/660 $\mathrm{nm}$. To this end, the patient's serum is first incubated with hydrochloric and sulfuric acids (reagent 1) in both cuvettes. Then, 3,5-dichlorophenyldiazonium tetrafluoroborate (reagent 2) is added to the color cuvette to produce azobilirubin. Finally, the blank cuvette absorbance is subtracted from the color cuvette absorbance to determine the net reaction absorbance $[4,5]$.

In this context, paraprotein interference is caused by precipitation of the particular paraprotein under the high acidic conditions used in the assay [3, 4], which has different consequences for the analytical result. First, absorbance of the blank cuvette does not parallel that of the color cuvette, and therefore, highly variable readings are obtained throughout the reaction [4]. Additionally, it has also been observed that interfered reactions often show very high absorbance values at the first photometric point in both cuvettes. Both facts may result in spurious D-Bil values [such as D-Bil values greater than total bilirubin (T-Bil) or negative/very low D-Bil values] but can also provide reasonable $\mathrm{D}$-Bil values.

There is no clear relationship between the concentration or isotype of the paraprotein and interference, which 
seems to be more dependent on the specific paraprotein than on the concentration. However, for samples of the same patient for which interferences were periodically detected, those were more prone to occur with high paraprotein concentrations only [6].

As recently described in a paper published by our group [6], if properly detected, this interference can be used to systematically detect patients with paraproteins who were not suspected a priori to have a monoclonal gammopathy, helping to advance diagnosis even for totally asymptomatic patients. In our previous paper, detection of interference relied on the Laboratory Information System (LIS) for detecting anomalous D-Bil results, as the $\mathrm{AU}$ instruments do not allow raising flags based on absorbance values [4]. However, and considering that paraprotein interference may also provide reasonable D-Bil results by mere chance, this protocol fails in detecting all paraprotein interferences and subsequent diagnosis of monoclonal gammopathies.

In this paper, an alternative method for detection of paraprotein interference on the D-Bil assay, regardless of the D-Bil result obtained, is investigated. This method is based on the revision of the photometric data, as was recently described for the T-Bil assay with another instrumentation [7].

\section{Materials and methods}

A total of 164 serum samples having paraproteins of different concentration (confirmed by immunofixation) were subjected to four replicate D-Bil measurements on the AU analyzers (Beckman Coulter, Brea, CA, USA). Interference was suspected when high relative standard deviation (RSD) values, together with one or more spurious D-Bil results (negative D-Bil, D-Bil above T-Bil) were obtained for any of the replicate measurements (see Table 1 for examples on interference identification). In any case, and to avoid misclassification of samples with D-Bil results close to the limit of detection $(0.14 \mathrm{mg} / \mathrm{L})$ for which fairly large \%RSD can be obtained, the presence of interference was finally confirmed for all samples with paraproteins by detection of turbidity after visual inspection of the reaction carried out manually, as described by Nauti et al. [3]. Note that in Table 1 we have included the actual value obtained for each replicate (even if it was below the limit of detection of $0.14 \mathrm{mg} / \mathrm{L}$ ), to illustrate the variability of results that can be obtained for these kinds of samples.

A total of 67 samples interfered the assay and were considered to be true positives (group 1). The remaining 97 samples containing paraproteins did not show interference and were considered true negatives (group 2). For comparison purposes, 2000 samples without paraproteins were also analyzed, including samples for which high absorbance background readings could also be expected (i.e. hemolyzed or lipemic samples). The samples analyzed included, in particular, 200 samples with hemolysis (group 3), 100 samples with lipemia (group 4), 100 samples with icterus (group 5) and 1600 samples free from hemolysis, lipemia, and icterus (group 6). These serum indexes were determined by routine photometric measurements.

Photometric data for all D-Bil reactions carried out in that set of samples were manually reviewed looking for an absorbance cutoff that best identifies the paraprotein interference. For this purpose, the first photometric point acquired in both cuvettes was evaluated at the different wavelengths available: 570 and $660 \mathrm{~nm}$ for the blank cuvette and $660 \mathrm{~nm}$ for the color cuvette. The absorbance at $570 \mathrm{~nm}$ in the color cuvette cannot be used for this purpose as it depends on the sample D-Bil concentration. Table 2 displays the descriptive statistics obtained for each sample group considered, and Figure 1 shows the same data graphically (for the 660-nm blank cuvette). Significant differences for the absorbance values were found between group 1 (interfering paraproteins) and the rest of the sample groups considered, which was proved by the corresponding Kruskal-Wallis test ( $p<0.01$ for all three wavelengths).

At a later stage, receiver operating characteristics (ROC) curves were used to establish the desired cut-off value. The process was repeated three times: (a) only with samples containing paraproteins (i.e. groups 1 and 2; population A), (b) with all sample groups (population B) and c) with all samples excluding those with hemolysis, icterus, and lipemia (i.e. groups 1, 2, and 6; population C). ROC curves were calculated with MedCalc (MedCalc Software BVBA, version 16.2.1, Ostend, Belgium) and compared using the method of DeLong et al. [8].

Once the optimal cut-off for probable interference was selected, all D-Bil reactions carried out in our laboratory during a period of 6 months (July-December 2015) were inspected, and those providing absorbance values above the cut-off were further investigated to try to discover paraproteins. Figure 2 is a graphical representation of this protocol.

The Hospital Universitario Miguel Servet (HUMS) Ethics Committee approved the study.

\section{Results}

Table 3 collects the results of the diagnostic efficiency parameters evaluated by ROC curves. As expected, the area under the curve (AUC) and the best possible cut-off depend on the population considered. There are not statistically significant differences between using 570 and $660 \mathrm{~nm}$ in the blank cuvette. This applies also to $660 \mathrm{~nm}$ in the color cuvette, except for population B. This set of samples includes an important percentage of hemolyzed or lipemic samples (the majority of false positives). In this case, a better diagnostic efficiency is obtained using the blank cuvette.

The final decision to monitor the absorbance at 570 or $660 \mathrm{~nm}$ in the blank cuvette was based on convenience. It has to be kept in mind that the cut-off value must be manually checked on the instrument display for each reaction carried out, and in this context, a round figure such as 0.0100 is much easier to visually identify. As shown in Table 3, the optimal cut-offs for populations A and B in 


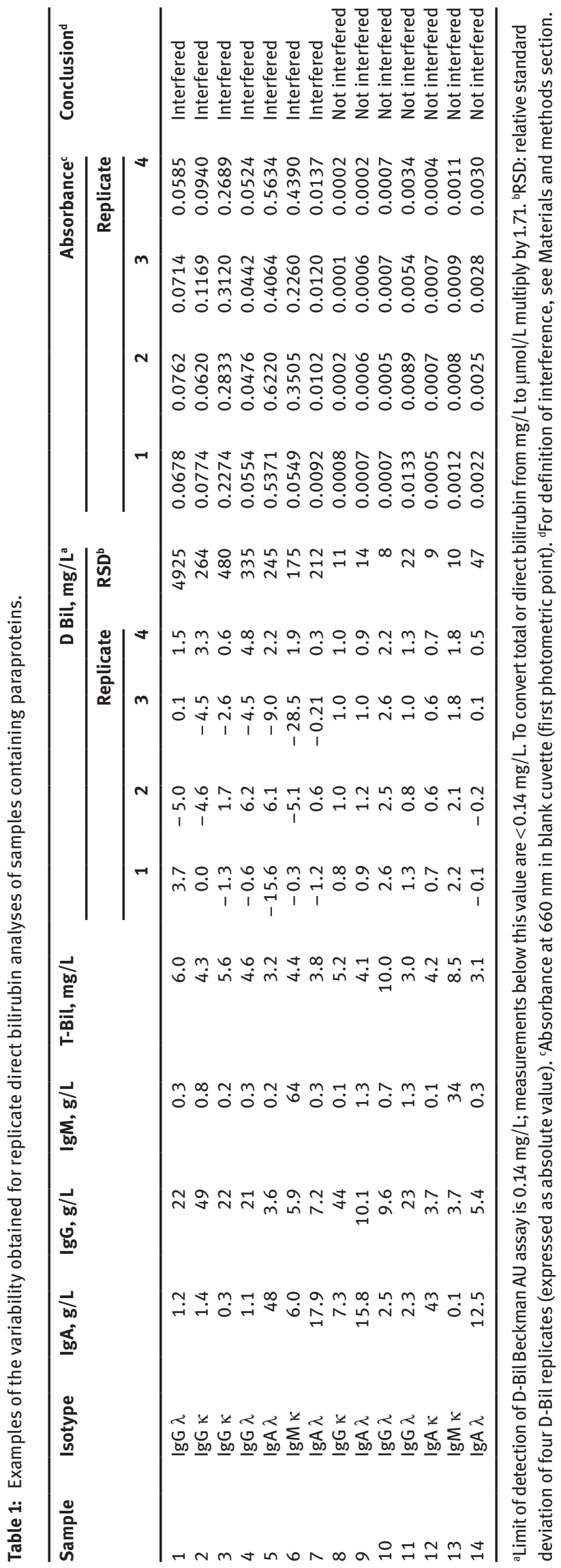

the blank cuvette at $660 \mathrm{~nm}$ are much closer to this round figure than those at $570 \mathrm{~nm}$, and hence, we decided to use the cut-off at $660 \mathrm{~nm}$ in the blank cuvette for further studies. Table 4 shows the diagnostic efficiency reached with this cut-off.

After the method was setup and during a period of 6 months, 15,181 D-Bil determinations were carried out in our laboratory and the corresponding photometric reactions were inspected, providing 331 measurements above the absorbance cut-off (see examples in Table 5); most of those measurements corresponded to samples with lipemia (85) or with intense hemolysis (186) and 53 were from patients already known to contain paraproteins or polyclonal immunoglobulins of elevated concentration. Finally, seven samples were further assayed to investigate the presence of paraproteins by immunoglobulin quantification, protein electrophoresis, and if necessary, immunofixation. In five of these samples, the presence of a paraprotein was confirmed, one showed elevated polyclonal immunoglobulins, and the remaining one did not show any altered parameter. The absorbance for this sample (sample 7 in Table 5) was just above the cut-off, and it is possible that, although not detected by the corresponding serum index measurement, it could be slightly lipemic.

\section{Discussion}

The presence of paraproteins may interfere with the D-Bil $\mathrm{AU}$ assay, and if it is properly detected, this interference may uncover non-diagnosed monoclonal gammopathies [6]. In this work we describe the protocol developed in our laboratory to detect this interference based on the observation that it often results in anomalous absorbance patterns. As the AU software does not automatically permit the raising of flags on photometric data, we developed a method based on manual revision of the absorbance values.

As explained in detail in the Materials and methods section, the best discriminating approach was based on selection of the optimal cut-off for the absorbance at the first photometric point in the blank cuvette. A great percentage of the samples analyzed in our laboratory on a daily basis belongs to newborns and are often hemolyzed. As a result, our typical set of samples would resemble population B in terms of false positives. For this population, the optimal cut-offs for the blank cuvette are 0.0102 and 0.0158 at 660 and $570 \mathrm{~nm}$, respectively, and selection of any of these cut-offs would provide similar results in 


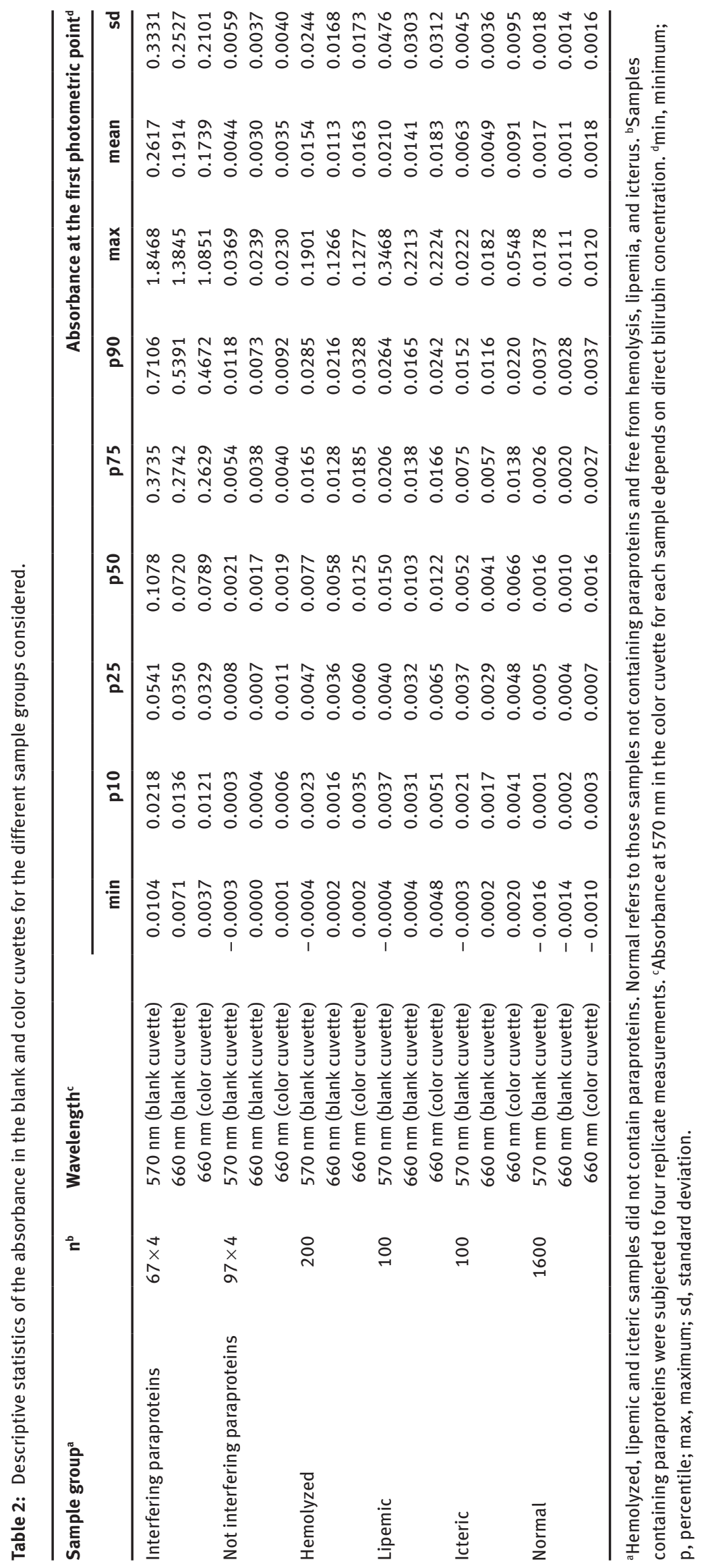




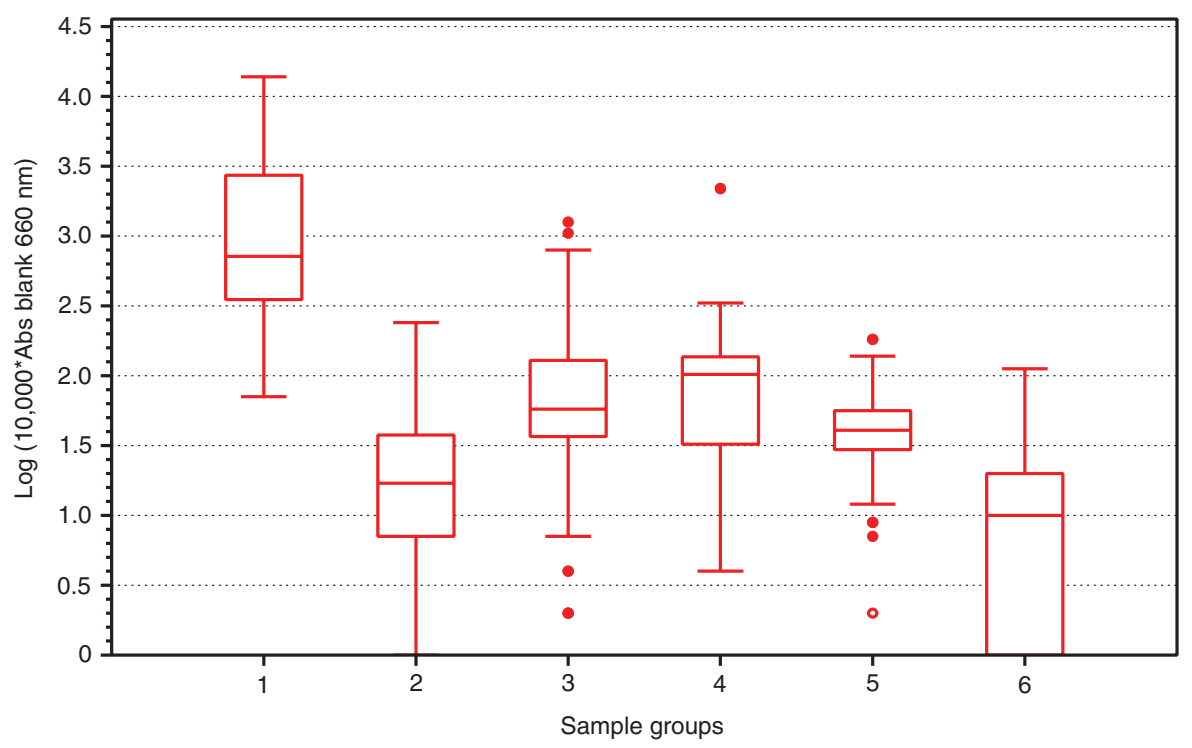

Figure 1: Absorbance values obtained at $660 \mathrm{~nm}$ in the blank cuvette (first photometric point) for the different sample groups considered. ${ }^{a}$

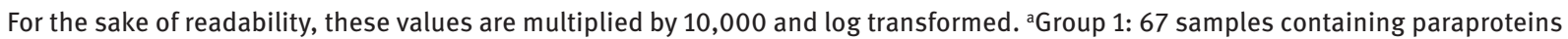
interfering with D-Bil Beckman AU assay; Group 2: 97 samples containing paraproteins not interfering with D-Bil AU assay; Group 3: 200 hemolyzed samples without paraproteins; Group 4: 100 lipemic samples without paraproteins; Group 5: 100 icteric samples without paraproteins; and Group 6:1600 samples without paraproteins and free from hemolysis, lipemia, and icterus.

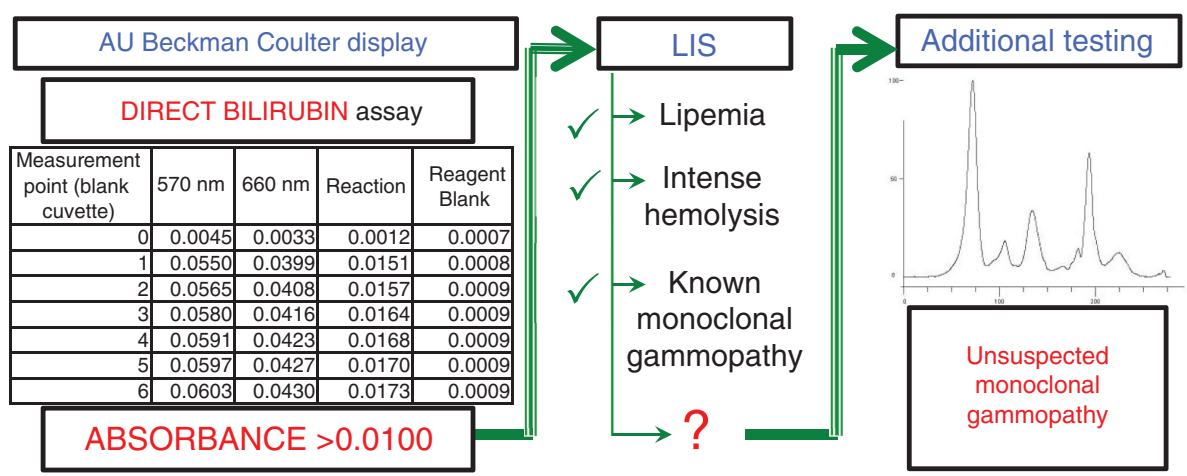

Figure 2: Graphical representation of the protocol developed in our laboratory to detect unsuspected monoclonal gammopathies.

Table 3: Diagnostic efficiency values for detecting interference by paraproteins in direct bilirubin (D-Bil) assay with different population samples.

\begin{tabular}{|c|c|c|c|c|c|}
\hline Population $^{a}$ & Cut-off ${ }^{b}$ & $A U C^{c}$ & p-Value ${ }^{d}$ & Sensitivity & Specificity \\
\hline \multirow[t]{3}{*}{ A: groups 1 and 2} & 1: Blank cuvette $(570 \mathrm{~nm}): 0.0160$ & 0.992 & Comparison 1-2: $p=0.51$ & 0.943 & 0.947 \\
\hline & 2: Blank cuvette $(660 \mathrm{~nm}): 0.0099$ & 0.993 & Comparison 2-3: $p=0.11$ & 0.955 & 0.947 \\
\hline & 3: Color cuvette $(660 \mathrm{~nm}): 0.0104$ & 0.986 & Comparison 1-3: $p=0.12$ & 0.920 & 0.916 \\
\hline \multirow[t]{3}{*}{ B: groups 1-6 } & 1: Blank cuvette $(570 \mathrm{~nm}): 0.0158$ & 0.991 & Comparison 1-2: $p=0.06$ & 0.943 & 0.947 \\
\hline & 2: Blank cuvette $(660 \mathrm{~nm}): 0.0102$ & 0.990 & Comparison $2-3: \mathrm{p}<0.01^{\mathrm{e}}$ & 0.943 & 0.937 \\
\hline & 3: Color cuvette $(660 \mathrm{~nm}): 0.0121$ & 0.979 & Comparison $1-3: \mathrm{p}<0.01^{\mathrm{e}}$ & 0.909 & 0.908 \\
\hline \multirow[t]{3}{*}{ C: groups 1,2 , and 6} & 1: Blank cuvette $(570 \mathrm{~nm}): 0.0116$ & 0.999 & Comparison $1-2: p=0.45$ & 0.989 & 0.988 \\
\hline & 2: Blank cuvette $(660 \mathrm{~nm}): 0.0079$ & 0.999 & Comparison 2-3: $p=0.11$ & 0.989 & 0.989 \\
\hline & 3: Color cuvette $(660 \mathrm{~nm}): 0.0073$ & 0.997 & Comparison 1-3: $p=0.10$ & 0.966 & 0.982 \\
\hline
\end{tabular}

${ }^{\mathrm{a}}$ See definition of groups in legend to Figure 1. ${ }^{\mathrm{b}}$ Best cut-off for absorbance at first photometric point $(570 \mathrm{~nm}$, reaction wavelength; $660 \mathrm{~nm}$, complementary wavelength. 'AUC: area under the curve. ${ }^{\text {d,e }}$ Significant $p$-value $<0.05$. 
Table 4: Diagnostic efficiency values for detecting interference by paraproteins on the D-Bil assay with different sample populations using 0.0100 as a cut-off for absorbance.

\begin{tabular}{llrr}
\hline Population $^{\text {a }}$ & Cut-off $=\mathbf{0 . 0 1 0 0}$ & Sensitivity & Specificity \\
\hline A: groups 1 and 2 & Blank cuvette $(570 \mathrm{~nm})$ & 1.000 & 0.890 \\
& Blank cuvette $(660 \mathrm{~nm})$ & 0.943 & 0.947 \\
& Color cuvette $(660 \mathrm{~nm})$ & 0.932 & 0.908 \\
B: groups 1-6 & Blank cuvette $(570 \mathrm{~nm})$ & 1.000 & 0.914 \\
& Blank cuvette $(660 \mathrm{~nm})$ & 0.943 & 0.936 \\
& Color cuvette $(660 \mathrm{~nm})$ & 0.932 & 0.888 \\
C: groups 1, 2, and 6 & Blank cuvette $(570 \mathrm{~nm})$ & 1.000 & 0.986 \\
& Blank cuvette $(660 \mathrm{~nm})$ & 0.943 & 0.994 \\
& Color cuvette $(660 \mathrm{~nm})$ & 0.932 & 0.991 \\
\hline
\end{tabular}

aSee definition of groups in legend to Figure 1.

terms of diagnostic efficiency. However, it has to be kept in mind that owing to the instrument limitations, photometric data must be manually reviewed on the instrument display, and in this situation, it is much easier to visually identify values exceeding a round figure such as 0.0100 . By selecting this cut-off, we decided to maximize specificity while maintaining a good sensitivity (Table 4), which allowed us to considerably reduce the number of results to be reviewed.

With this working protocol and during a period or 6 months, 15,181 D-Bil determinations were reviewed and 44 samples interfered by paraproteins were detected; five of these samples corresponded to patients unsuspected of having a monoclonal gammopathy, while the rest were from patients already diagnosed with different types of monoclonal gammopathies, most of them followed up at the Hematology Department of the HUMS.

Practical implementation of the method developed consisted of the daily review of all of the D-Bil reactions carried out at the laboratory the morning after they were measured. Those samples with absorbance values above the selected cut-off were subsequently reviewed on the LIS to confirm whether they were samples with lipemia or intense hemolysis or whether they belonged to patients already known to contain paraproteins or elevated polyclonal immunoglobulins. Only samples outside these categories were further assayed to determine if a paraprotein was present. The whole process of reviewing the D-Bil photometric data and deciding which samples should call for additional testing only required $10-15 \mathrm{~min}$ of additional work.

The method developed allowed us to confirm that in a great percentage of the reactions, the presence of interference by paraproteins still provides totally reasonable D-Bil results ( $43 \%$ in our case). For this reason, relying on the LIS for flagging spurious D-Bil results only (D-Bil greater than the T-Bil or negative/very low D-Bil results) would result in many cases of monoclonal gammopathies not properly detected. In this particular study three out of the five patients first discovered to have a monoclonal gammopathy (samples 6 and 8 in Table 5) would have been missed.

Table 5: Examples of samples that provided reasonable direct bilirubin (D-Bil) results with absorbance readings above the selected cut-off.

\begin{tabular}{|c|c|c|c|c|c|c|c|c|c|c|}
\hline \multirow[t]{3}{*}{ Sample } & \multirow{3}{*}{$\begin{array}{r}\text { Total } \\
\text { bilirubin, } \\
\mathrm{mg} / \mathrm{L}^{\mathrm{a}}\end{array}$} & \multirow{3}{*}{$\begin{array}{r}\text { Direct } \\
\text { bilirubin, } \\
\mathrm{mg} / \mathrm{L}^{\mathrm{a}}\end{array}$} & \multicolumn{4}{|c|}{ Absorbance $^{b}$} & \multirow[t]{3}{*}{ Comment $^{c, d}$} & \multicolumn{3}{|c|}{$\operatorname{Ig} A, g / L \lg G, g / L \operatorname{Ig} M, g / L$} \\
\hline & & & \multicolumn{2}{|c|}{ Blank cuvette } & \multicolumn{2}{|c|}{ Color cuvette } & & & & \\
\hline & & & $570 \mathrm{~nm}$ & $660 \mathrm{~nm}$ & $570 \mathrm{~nm}$ & $660 \mathrm{~nm}$ & & & & \\
\hline 1 & 183 & 6.2 & 0.0299 & 0.0207 & 0.0298 & 0.0215 & Intense hemolysis. Newborn. & & & \\
\hline 2 & 3.4 & 1.0 & 0.3468 & 0.2212 & 0.3477 & 0.2210 & Intense lipemia & & & \\
\hline 3 & 7.5 & 2.1 & 0.0353 & 0.0220 & 0.0148 & 0.0051 & Known paraproteinemia (MGUS IgA א) & 10.0 & 25 & 0.7 \\
\hline 4 & 8.2 & 4.7 & 0.2943 & 0.2132 & 0.3124 & 0.2227 & Known paraproteinemia (MM IgG к) & 2.4 & 35 & 0.3 \\
\hline 5 & 24.8 & 6.6 & 0.0229 & 0.0141 & 0.0340 & 0.0166 & Chronic elevated polyclonal immunoglobulins & 3.9 & 31 & 3.7 \\
\hline 6 & 4.0 & 0.6 & 0.3110 & 0.2285 & 0.3126 & 0.2293 & $\begin{array}{l}\text { Further assayed. Monoclonal gammopathy } \\
\text { IgG } \kappa \text { confirmed ( } 82 \text {-year-old woman under } \\
\text { evaluation. MM suspected.) }\end{array}$ & 0.7 & 22 & 0.8 \\
\hline 7 & 11.4 & 3.0 & 0.0183 & 0.0113 & 0.0248 & 0.0141 & Further assayed. Paraprotein not present. & & & \\
\hline 8 & 5.6 & 4.5 & 0.1321 & 0.0892 & 0.1655 & 0.1073 & $\begin{array}{l}\text { Further assayed. Monoclonal gammopathy } \\
\text { IgG } \kappa \text { confirmed ( } 60 \text {-year-old woman under } \\
\text { evaluation). }\end{array}$ & 2.0 & 22 & 0.9 \\
\hline
\end{tabular}

${ }^{\mathrm{a}}$ To convert total or direct bilirubin from $\mathrm{mg} / \mathrm{L}$ to $\mu \mathrm{mol} / \mathrm{L}$ multiply by 1.71 . ${ }^{\mathrm{b}} \mathrm{Absorbance}$ at first photometric point. A value $>0.0100$ at $660 \mathrm{~nm}$ in blank cuvette requires investigation. 'Further assayed refers to samples not hemolyzed or lipemic but suspected to contain paraproteins from patients unknown to have monoclonal gammopathies or elevated polyclonal immunoglobulins were further investigated. ${ }^{\mathrm{d}} \mathrm{MGUS}$, monoclonal gammopathy of undetermined significance; MM, multiple myeloma. 
Although moving from only flagging unreasonable/ non-possible D-Bil results to review all the D-Bil photometric reactions has been a considerable improvement in the protocol set up in our laboratory, there is evidence that supports the conclusion that to detect all samples interfered by paraproteins, monitoring photometric reactions for all chemistry assays would be recommended. In this sense, for instance, Alberti et al. [9] have recently reported a particular patient paraprotein precipitating at both extremely high and extremely low $\mathrm{pH}$ values, implying that all assays carried out under these conditions, specifically the D-Bil, creatinine and total protein AU assays, would be interfered. As the composition of each paraprotein is patient dependent, each sample containing a paraprotein may show a particular $\mathrm{pH}$ at which it aggregates, and therefore, the assay(s) affected for a particular patient can be different from those affected for other patients. As a result, it seems clear that the best way to maximize possibilities for interference detection would be to monitor all photometric reactions for all chemistry assays.

The particularities of the method presented in this work are a good illustration of this issue. In fact, most laboratories only perform the D-Bil assay when the T-Bil result is above the upper reference limit, and hence, the sole application of this method would be clearly insufficient for detecting paraproteins in most cases. In our particular study, none of the five patients discovered to have an unsuspected paraprotein would have been detected if D-Bil analysis had been only performed for samples for which the T-Bil result was above the upper reference limit.

Finally, it may be worth mentioning that our main goal with this protocol, in addition to detecting interferences that may translate into erroneous test results, was to discover the presence of unsuspected paraproteins. To systematically search for information not ordered by the clinician could raise ethical questions [10, 11], and the answers to these questions may vary depending on the country. However, as our laboratory is part of the public Spanish Health System, this fact was not really an issue in our case. Furthermore, all samples considered in this study belonged to patients whose practitioner had requested a blood test to assess their general health status. Therefore, revealing the presence of large paraproteins can only bring benefits to the patient, even though the clinician had not specifically requested such tests. For patients presenting with paraproteins, it is of paramount importance to begin evaluation and/or treatment (if required) as early as possible, especially considering that most of the patients exhibiting this interference present paraproteins of high concentration [6]. Even if asymptomatic, these patients should be checked periodically, especially considering that annual progression to malignancy for monoclonal gammopathy of undetermined significance (MGUS) is as high as $1 \%[12]$.

\section{Conclusions}

We have demonstrated that reviewing the D-Bil photometric data is a valuable strategy that serves to systematically detect interference by paraproteins on the D-Bil AU assay, regardless of the particular D-Bil result. However, the AU software requires that each reaction be manually reviewed on the instrument display, without the possibility of automatically flagging anomalous absorbance patterns.

As paraproteins are patient-dependent, and each specific paraprotein may precipitate at a particular $\mathrm{pH}$, different assays may be affected for different patients. Therefore, all chemistry reactions should be monitored to try to detect interference in all samples, maximizing the possibilities to diagnose monoclonal gammopathies. This will only be possible when current instrumentation is supplied with an automated flagging system that would allow monitoring the tens of thousands of reactions carried out at clinical laboratories on a daily basis.

Acknowledgments: The authors acknowledge the funding from CTQ2015-64684-P (MINECO/FEDER) and from the Aragón Government (Fondo Europeo de Desarrollo Regional).

Author contributions: All the authors have accepted responsibility for the entire content of this submitted manuscript and approved submission.

Research funding: CTQ2015-64684-P (MINECO/FEDER), Aragón Government (Fondo Europeo de Desarrollo Regional).

Employment or leadership: None declared.

Honorarium: None declared.

Competing interests: The funding organization(s) played no role in the study design; in the collection, analysis, and interpretation of data; in the writing of the report; or in the decision to submit the report for publication.

\section{References}

1. Bakker AJ, Muecke M. Gammopathy interference in clinical chemistry assays: mechanisms, detection and prevention. Clin Chem Lab Med 2007;45:1240-3.

2. Dalal BI, Brigden ML. Factitious biochemical measurements resulting from hematologic conditions. Am J Clin Pathol 2009;131:195-204. 
3. Nauti A, Barassi A, Merlini G, d’Eril GV. Paraprotein interference in an assay of conjugated bilirubin. Clin Chem 2005;51:1076-7.

4. Yang Y, Howanitz PJ, Howanitz JH, Gorfajn H, Wong K. Paraproteins are a common cause of interferences with automated chemistry methods. Arch Pathol Lab Med 2008;132:217-23.

5. Song L, Kelly KA, Butch AW. Monoclonal and polyclonal immunoglobulin interference in a conjugated bilirubin assay. Arch Pathol Lab Med 2014;138:950-4.

6. García-González E, González-Tarancón R, Aramendía M, Rello L. Analytical interference by monoclonal immunoglobulins on the direct bilirubin AU Beckman Coulter assay: the benefit of unsuspected diagnosis from spurious results. Clin Chem Lab Med 2016;54:1329-35.

7. Seimiya M, Suzuki Y, Yoshida T, Sawabe Y, Matsushita K, Nomura F. The abnormal reaction data-detecting function of the automated biochemical analyzer was useful to prevent erroneous total-bilirubin measurement and to identify monoclonal proteins. Clin Chim Acta 2015;441:44-6.

8. DeLong ER, DeLong DM, Clarke-Pearson DL. Comparing the areas under two or more correlated receiver operating characteristic curves: a nonparametric approach. Biometrics 1998;44:837-45.

9. Alberti MO, Drake TA, Song L. The pH of chemistry assays plays an important role in monoclonal immunoglobulin interferences. Pract Lab Med 2015;3:8-16.

10. McQueen M). Some ethical and design challenges of screening programs and screening tests. Clin Chim Acta 2002;315:41-8.

11. Wijeratne N, Benatar SR. Ethical issues in laboratory medicine. J Clin Pathol 2010;63:97-8.

12. Kyle RA, Rajkumar SV. Monoclonal gammopathies of undetermined significance. Best Pract Res Clin Haematol 2005;18:689-707. 